\title{
Prevention of Drug-Induced Risks
}

\author{
Deborah Szafir, ${ }_{1}^{1}$ Hervé Lelouët, ${ }^{2}$ Jean-Louis Imbs ${ }^{3}$ and the Participants in Round Table No. 3, \\ Giens XVIII ${ }^{\dagger *}$ \\ 1 Laboratoire Roche, Neuilly-sur-Seine, France \\ 2 CHU Henri Mondor, Créteil, France \\ 3 Service des Maladies Vasculaires et Pharmacologie Clinique, Hôpitaux Universitaires de Strasbourg, Strasbourg, France
}

\begin{abstract}
Effective prevention of drug-induced risks depends on an accurate understanding of their triggering or predisposing factors, and the quality of information on these available to prescribing practitioners and users. All preclinical and clinical data available on the proprietary medicinal product concerned should facilitate identification of a risk, and these data should be compared with existing data on drugs sharing the same mode of action or therapeutic strategy. This information should be based on a communication plan adapted to the context of the disease under treatment, the therapeutic alternatives available and the benefits expected.
\end{abstract}

Keywords: iatrogenicity, drugs, prevention, information

Text received May 20, 2003; accepted June 4, 2003

Only recognised risks, for which the causal factors have been analysed, can be prevented. For this reason, the round-table discussion chose to consider the two components essential to any preventive strategy: identification of the risk associated with the use of a medicinal product, and communication aimed at its optimum management. Bias, random events during therapy, legal aspects, the management of media-induced crises, and application of the principle of precaution were not covered during the discussion.

\section{Identification of Drug-Induced Risks: Pathways to be Explored}

An unexpected adverse event is rarely identified during the formal stages of drug development. Its first appearance is often during the clinical phase, and its analysis will pass through four stages: (i) observation of the risk; (ii) validation of the danger; (iii) implementation of a preventive procedure; and (iv) assessment of the efficacy of the measures implemented. The role played by preclinical research into possible prolongation of the QT interval on an electrocardiogram to prevent arrhythmia asso- ciated with changes in cardiac electrophysiology is a recent example demonstrating the advances that have been achieved.

\section{1 The Preclinical Setting}

Although safety pharmacology ${ }^{[1-3]}$ has seen considerable progress in terms of efficacy and logistics, its results should be better targeted to objectives concerning the safe use of a drug: definition of the population(s) affected by a therapeutic strategy; and identification of individuals (or a population) who are at risk of presenting with the adverse effect. This transversal approach goes beyond the conclusions of a pharmacology report in an attempt to integrate them into a global understanding of the drug. If advances in genomics can succeed in detailing the relationships between the genetic code and organ functions, they will play a major role in this respect. Genomic toxicology is perhaps closer to the target when it envisages setting up 'molecular signature' banks to record the cellular effects of toxic compounds, which will then serve as references to determine and predict the toxicity of a potential new drug.

$\dagger$ For a list of participants, please see the end of the article.

* Articles, analyses and proposals arising from the Giens Clinical Pharmacology Meetings are the responsibility of their authors and do not reflect the views held by their supervisory organisation. 


\subsection{Clinical Use Prior to Obtaining Marketing Authorisation}

Once again, emphasis is laid upon the value of a global view of the safety data available, and their transversal exploitation in order to analyse their relationship with preclinical findings. Thus, any analyses of logically related biological data should include variations in the ranges of normal values and include significant clinical findings.

Such analyses are already undertaken by those responsible for developing new drugs. However, systematic meta-analyses are still required to examine these parameters for drugs developed within a pharmacological family, or for compounds likely to exhibit adverse effects caused by common mechanisms. Since these may they be carried out within a pharmaceutical company only in exceptional circumstances, they need to be considered by the regulatory authorities. The biological and clinical profiles of similar medicinal products should be compared, whereas such comparisons presently originate only from the recollections and experience of experts. Such meta-analyses could constitute the reference values for control groups, depending on the populations treated and based on the incidence of biological abnormalities in placebo-treated individuals observed during the different studies included in registration records. This approach could provide a warning in an analogous population during the development of a new product. Similarly, these analyses could improve the safety of healthy volunteers participating in clinical studies.

\subsection{Clinical Use During the Postmarketing Phase}

The role of pharmacovigilance units in pharmaceutical companies, and of regional pharmacovigilance centres (centres régionaux de pharmacovigilance [CRPV]), is obviously crucial. The spontaneous notification of adverse events is recognised as the most effective and least costly monitoring method. It should now be supplemented by pharmacological-epidemiological monitoring to check the suitability of the target population of a medicinal product, as defined in the Summary of Product Characteristics (SPCs). Social security organisations (Caisse Nationale d'Assurance Maladie [CNAM], Caisse Nationale d'Assurance Maladie des Professions Indépendantes [CANAM]) are now able to compare the delivery and prescribing of drugs and the compliance of medical practitioners with good prescribing practices. Thus, in 1999, the CANAM carried out a survey on how far prescribing practitioners took account of known drug interactions. Among other results, this survey recognised the influence of a personalised letter sent to the practitioner, asking him to specify the precise reasons for a prescription, and asking for his comments on them. This condition for satisfactory efficacy should certainly be exploited to inform prescribing practitioners about the correct use of medicinal products. ${ }^{[4]}$

\section{Communicating the Risks Associated with Medicinal Products}

The group decided not to review the information provided in the SPCs or package leaflets of drugs, nor to consider the reasons why insufficient information is provided during the teaching of pharmacology and therapeutics. It focused its efforts on analysing a communication plan for public health purposes or, more precisely, on new information arising out of the detection of an adverse drug effect. In the first instance, major differences can be seen between those providing information (regulatory authorities, the pharmaceutical industry, specialised publications, or media aimed at the public) and their target audiences (patients, their families, healthcare professionals and, if relevant, the judgement). The degree of freedom allowed for communication differs, depending on the target audience and those disseminating the information. Thus, information provided by the pharmaceutical industry is usually limited to the contents of the SPC or the text of a letter approved by the regulatory authorities.

The aim is to retain the confidence of society, to educate the public and increase its awareness of personal responsibilities, and to reduce avoidable morbidity and mortality. The means must be made available to assess the impact of an 'advertising' approach on these objectives. ${ }^{[5]}$

The correct timing of a communication is the appearance of new facts that may modify the risk/benefit ratio, and thus require a change in the mode of prescribing of a medicinal product. Communication is essential when rumours or poorly controlled information is spread. Assessment of the level of certainty required before any communication is made must be based on a judgement weighing the potential seriousness of the risk against the level of acceptance by the public. The setting up of a committee of citizens within the National Institute of Clinical Excellence (NICE) in the United Kingdom, aimed at taking account of public viewpoints in any decision-making process, will no doubt enable practical recommendations to be made in this respect.

The definition of a communication plan and its targets is a high priority. Information must be clearly expressed, based on practical experience, and be placed in the context of the disease under treatment, the available therapeutic alternatives, and the expected benefits. Exchanges with media professionals (infrequent at present) need to be organised: the choice of approaches adapted to different audiences should benefit as a consequence. 


\section{Proposals for Action}

\subsection{New Ways to Identify Drug Risks}

- Profit from the analysis of logical associations of laboratory results, obtained during preclinical development or clinical use, to detect (prior to marketing) any risks of toxicity or sensitisation.

- In the real-life setting of clinical studies, explore the possibilities of using genomic techniques to identify high-risk patients, and to validate the tests proposed for practical use.

- Build up a safety register encompassing all the information available on both single compounds and all compounds in the same pharmacological family.

\subsection{Communicating Drug Risks}

- Encourage exchanges with journalists' associations in order to reach a mutual understanding about the language preferred in this type of communication.

- Define the optimum moment for communication.

- Define the procedures needed to draw up a stepwise communication plan including all partners and audiences: who communicates to whom, and in what order?

\section{Participants}

R. Barouki (INSERM U490, Paris), J.P. Blayac (CRPV, Montpellier), B. Bons (GlaxoSmithKline, Marly-le-Roi), J. Caron (CRPV, Lille), A. Castot
(Afssaps, Saint-Denis), M. Childs (Mediscan, Paris), V. Daurat (DRC, Paris), J. Deligne (CANAM, Lille), C. Delmas (Roche, Neuilly-sur-Seine), J.P. Demarez (Biomérieux-Pierre Fabre, Boulogne), E. Eschwege (INSERM U21, Villejuif), A. Garcia-Gibot (LEEM, Paris), F. Girard (Sanofi-Synthélabo, Le Plessis-Robinson), J. Guilhot (CNAMTS, Paris), C. Kreft-Jaïs (Afssaps, Saint-Denis), G. Lagier (CRPV, Paris), V. Lamarque (Novartis, RueilMalmaison), B. Lebrun-Vignes (Hôpital Pitié Salpétrière, Paris), A. Lelouet (HEGP, Paris), P. Maillère (Servier, Courbevoie), J.L. Malvy (MGVM Consultants, Paris), M. Mazraani (Hôpital St-Vincent de Paul, Paris), L. MoachonChauvelot (Hôpital Cochin, Paris), D. Muzard (Lilly, Saint-Cloud), P. Olivier (CRPV, Toulouse), M. Pappo (GSK, Marly-le-Roi), E. Pastor (AJMED, Paris), C. Riché (CRPV, Brest), D. Sallière (Sanofi-Synthélabo, Bagneux), L. Thomas (CRPV, Créteil).

\section{References}

1. Barbier A, Caille D. La pharmacologie de sécurité : une réalité réglementaire. Implications. Thérapie 2002; 57: 104-8

2. Redfern WS, Wakefield ID, Prior H, et al. Safety pharmacology: a progressive approach. Fundam Clin Pharmacol 2002; 16: 161-73

3. 14e Journée d'étude de l'association pour le développement de la pharmacologie clinique : de l'étude de la tolérance à la sécurité d'emploi. Lettre Pharmacologue 2002; 16: 66-78

4. Vogt EM. Effective communication of drug safety information to patients and the public: a new look. Drug Saf 2002; 25: 313-21

5. Tubiana M, Legrain M. Comment développer et améliorer les actions de prévention dans le système de santé français ? Bull Acad Natl Med 2002; 186: 447-540

Correspondence and offprints: Deborah Szafir, Worldwide Affiliates Operation \& EMEA European Coordinator for Pharmacovigilance, Produits Roche, 52 Boulevard du Parc, 92521 Neuilly-sur-Seine, France.

E-mail: deborah.szafir@roche.com 